\title{
Practical aspects of increasing workers efficient work time in a high noise level industrial area
}

\author{
R. Mikalauskas*, V. Volkovas** \\ *Kaunas University of Technology, Technological System Diagnostic Institute Kęstučio str. 27, 44312 Kaunas, Lithuania, \\ E-mail: robertas.mikalauskas@ktu.lt \\ **Kaunas University of Technology, Technological System Diagnostic Institute Kęstučio str. 27, 44312 Kaunas, \\ Lithuania, E-mail: vitalijus.volkovas@ktu.lt
}

cross $^{\text {ref }}$ http://dx.doi.org/10.5755/j01.mech.21.6.13240

\section{Introduction}

In the modern world there exists a lot of negative impacts on human health, including acoustic noise in a technical environment surrounding the individual. That noise is usually being emitted by various equipment at a workplace, outside or in a household.

Today the problem of fighting the noise is widely discussed; it is named as priority in order to ensure human safety and health, appropriate work environment and prevention of professional diseases.

The base of human protection from the risk due to noise in many European countries is on the number of European Parliament and Council Directives: 2000/14/EC, 2003/10/EC, 2002/44/EC, the occupational exposure standards of the countries [1-3]. These requirements impelled two directions of studies in solving the industrial noise problem and human health protection: identification and assessment of acoustic fields; environmental acoustic field control and insurance of optimal working conditions for the personnel. The first direction focuses more on acoustic fields modelling $[4,5]$ and prediction, where the second direction focuses on creating means and tools [4] for reducing noise levels, as well as their implementation outdoors [6,7], in buildings [8] and industrial environment. Research in the aforementioned second direction and other known methods for reducing nose create a path for studying the possibility of increasing staff work time until a permissible noise exposure dose is accumulated. The effectiveness of such methods can be evaluated with an effective work time $T_{e}$ which characterises the amount of time a staff member can work in a noisy environment so that the noise exposure value $L_{E X, 8 h}=85 \mathrm{~dB}(\mathrm{~A})$ [1] is not exceeded. This paper studies a group of noise sources that generate high level (up to $110 \mathrm{~dB}$ ) level noise - specific acoustic fields in an enclosed space, as well as methodology for effectively increasing the work time in such environment until a permissible daily noise exposure dose is accumulated.

The investigation of acoustic fields, generated by various sources is a specific and complex problem, which needs application of complex solutions that are presented in this paper as practical methodology for increasing time duration, until a permissible noise doze for workers would be achieved. To characterize and define this problem a number of questions were answered. Among them we can mention an analysis of acoustic fields' specific features that helps us to designate a corresponding investigation and create practical methodology for enclosed spaces with wideband acoustic field control generated from multiple sources for the aim of efficient and practical noise reduction in the denoted place.

While solving various aspects of the acoustical problem, many researches have been done [9-13], but there is a lack of systematic view, to suggest general investigation methodology, which could be used to evaluate specific characteristic of the acoustic field and would allow to achieve an efficient and economically competitive solution for increasing the time duration until an allowable noise dose for workers will be reached.

For that purpose an optimization analysis was conducted by using a limited number of experimental data as well as modelling the acoustic power and directivity for some noise sources. With the help of this analysis, the acoustic field in an enclosed space with a complicated acoustic excitation was successfully simulated and an optimal passive noise reduction screen system was selected. The noise passive reduction results $\sim 5-6 \mathrm{~dB}$ were the same as achieved from modifying the structures of some working units responsible for the noise level [14].

This paper investigates specific acoustical fields in systematic point of view that are generated by several turbine compressors in an enclosed space as well as general methodology of reducing noise levels in a denoted place of an industrial shop room. Practical aspects for applying this approach are connected with increasing the staff work time until a maximum accumulated noise exposure dose is reached, by more than six times.

\section{Principles of acoustic field control and measurement quantities}

Efficient noise reduction in industrial premises is possible only by assessing the problem from systematic positions. The formulation of noise control strategy and application of acoustic field's measurement for such premises requires particular steps. According the requirements of the series of standards ISO 11690 [15-17] they can be described as:

a) formulation of purposes and criteria;

b) evaluation and identification of noise;

c) noise control;

d) composition of control program;

e) use of measurements;

f) evaluation of noise reduction efficiency.

The sound noise control can be distinguished into two levels as illustrated in Fig. 1. 


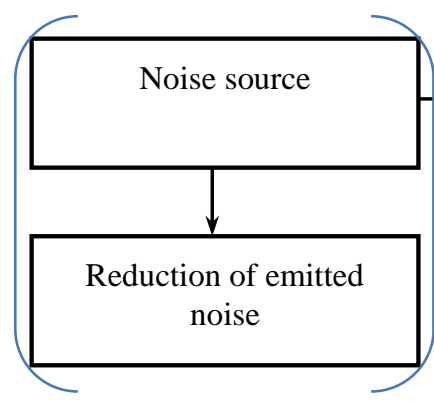

(Primary noise control)

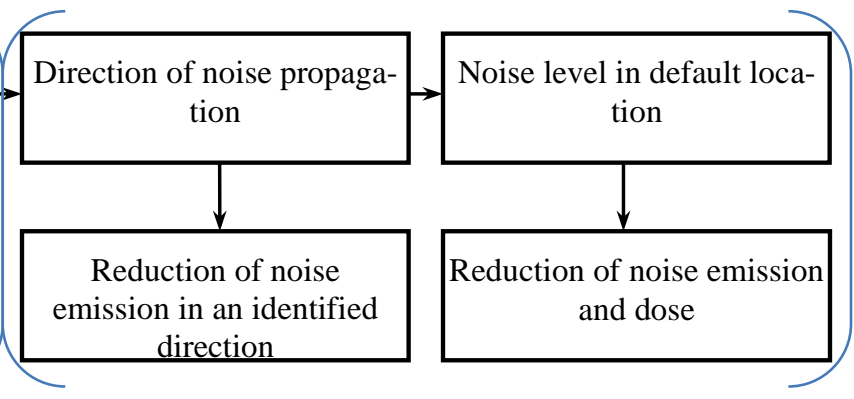

(Secondary noise control)

Fig. 1 Levels of noise control

In a general practical situation we have noise sources that can be identified and impacted only so that there would be no changes in the construction, negative impacts on technological process and suggested solutions would not require large capital investments. In this work it was investigated how and by what principles it is possible to control and purposefully reduce emitted acoustic field level in compressors room premises with 8 high output turbo compressors (Fig. 2).

The mentioned European directives define the measurement quantities of acoustic fields, that is: $A$ - weighted average time acoustic noise pressure $L_{A e q}$ and $C$ - weighted maximum (peak) sound presser level $L_{C p k}$. Thus in practice methodically it is correct to measure these quantities, or if the purpose requires measuring another quantity, the results still can be provided as $L_{A e q}$ and $L_{C p k}$. This is necessary when the intensimetry is applied to the measurements, which allows determining the intensity and propagation direction of the sound. But the results of the methods application for comparative analysis still have to be delivered by mentioned quantities.

The sound pressure level measurements are useful if the measurements can be made for one or for at least part of sources. While localising in sources particular emitted noise points, it is purposeful to use intensimetry.

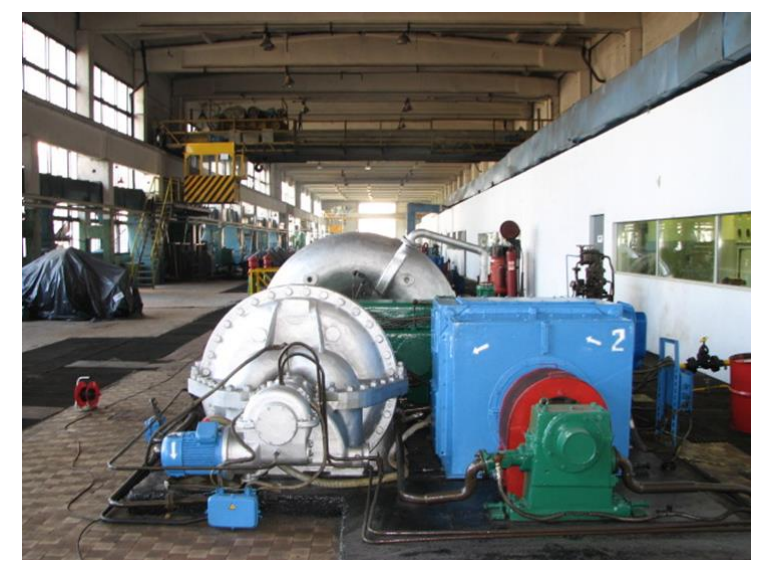

a
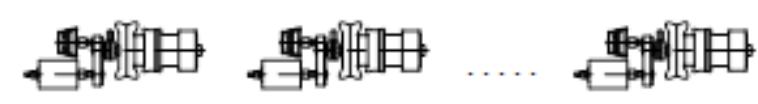

GTT-3 No. 1 GTT-3 No. 2

GTT-3 No. 8 compression room

$\mathrm{b}$

Fig. 2 Arrangement of turbo compressors (a) in (b) compression room premises
For the measurement of the mentioned features of the acoustic field parameters and analysis B\&K (Denmark) sound pressure meter 2260 Investigator, equipment Pulse $3600 \mathrm{C}$ and sound intensity microphone antenna of type 3599 were used.

\section{The stages for reduction of sound pressure level in closed space}

The general procedure for sound pressure level reduction can be explained by below presented scheme (Fig. 3), where using systematic approach main stages are distinguished.

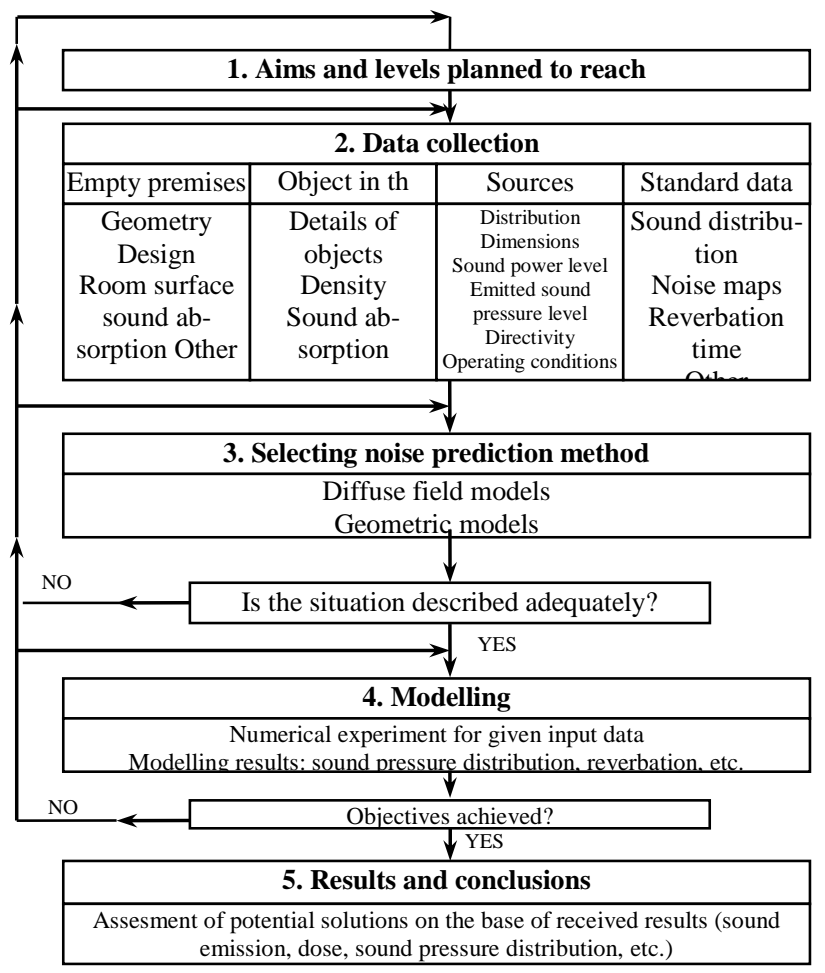

Fig. 3 Overall procedure of sound pressure level reduction

The reduction of noise, in the limits of systematic understanding requires data accumulation and investigation of specificity of acoustic fields, composition of mathematical models and modelling of acoustic fields, result analysis and decision making. This would be quite universal way both for formulation of the purpose and naming aimed sound pressure level we have to investigate the nature of acoustic field and define its specific features. The obtained results can be used for the formulation of protective means principal solutions, selection of sound absorbing material, 
numerical modelling of acoustic fields and physical protective means and optimization of constructional solutions. In our opinion, this direction is most promising for enhancing operating conditions in a particular space (compression room premises) and to increase efficient work time $T_{e}$ for workers who are repairing one of the turbo compressors in the mentioned compression room. Then we need to describe some specific aspects of problem solution direction and the potential effect of procedure realization.

\subsection{Analysis of noise nature}

The nature of the noise was determined by measuring the change of acoustic noise parameters in time. With the help of particular examples of turbo compressors in compression room we will show how noise nature assessment procedure is applied.

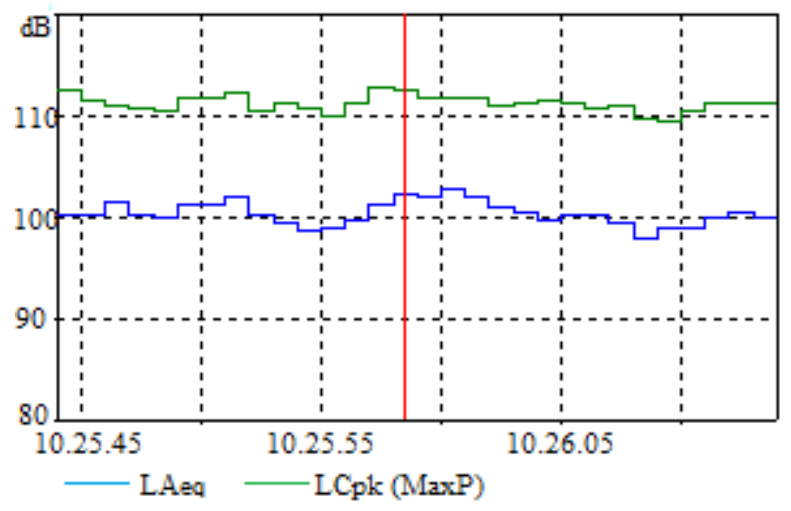

Fig. 4 Variation of the time - averaged sound pressure level

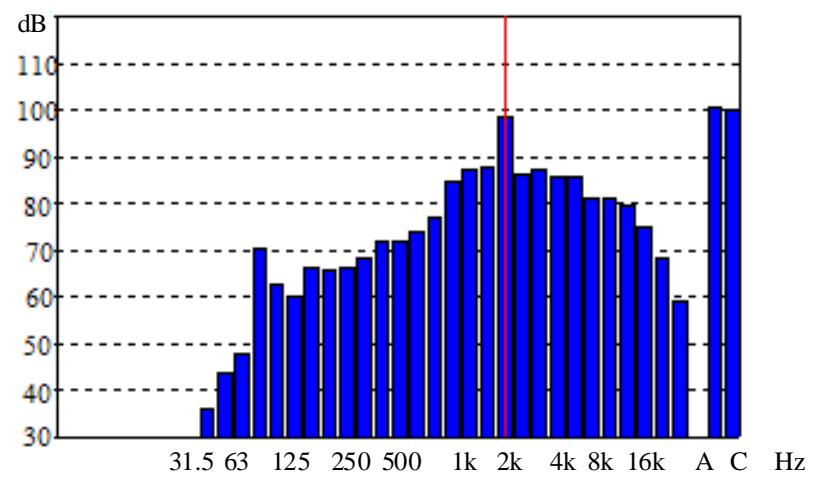

Fig. 5 Characteristic spectrum of the time - averaged sound pressure level in1/3 octave frequency range

The noise was measured in the height of $1.5 \mathrm{~m}$ from the floor of turbo compressor turbine zone (about $3.5 \mathrm{~m}$ from the end of the turbo compressor) within $1 \mathrm{~m} \ldots$ $1.3 \mathrm{~m}$ distance from the aggregate. The measurement quantities were: A-weighted average time acoustic noise sound pressure level $L_{A e q}$ and C-weighted maximum (peak) sound pressure level $L_{C p k}$. The results of the measurements are provided in Figs. 4 and 5.

The Fig. 4 presents variation of equivalent sound presser level parameters $L_{A e q}$ and $L_{C p k}$ in time. The $1 / 3$ octave characteristic spectrum of acoustic noise is provided in Fig. 5. Using Figs. 4 and 5 and definitions of hygiene norms [3] the nature of noise emitted by turbo compressors in the workshop room can be defined as constant broadband noise.

\subsection{Estimation of extreme acoustic field criteria}

The measurements of turbo compressor emitted noise intensity for the investigation of the features of noise sources acoustic field were made. To determine turbo compressor's emitted noise acoustic field extreme zones the separate nodes emitted noise was measured. In order to localize extreme zones as precisely as possible, the measurements were made within $0.25 \mathrm{~m}$ distance from investigated node surface. The noise of turbo compressor was measured in five longitudinal sections.

Using intensimetry principle the noise emitted by turbo compressor and distribution pipes were applied. Fig. 7 presents the turbo compressor's measured surface involute (without right plane). The Selected dimensions for measured surface segments were $1 \mathrm{~m} \times 1 \mathrm{~m}$.

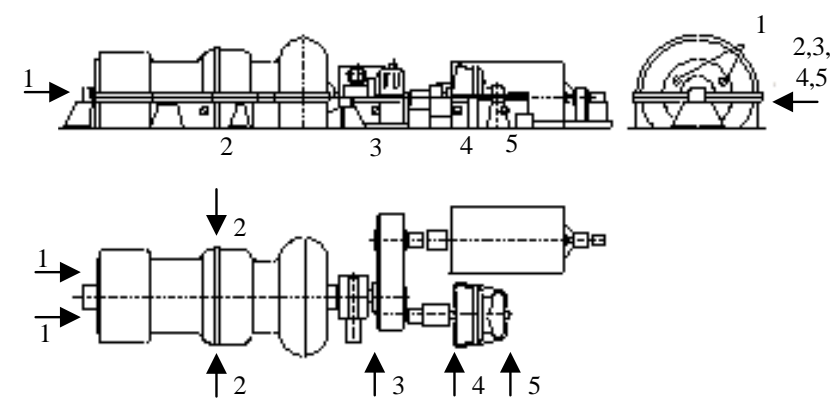

Fig. 6 Turbo compressor's maximum noise emission zones

When analysing measurement results, the maximum noise emission zones were determined. In Fig. 6 these zones are indicated by arrows:

- at the axial compressor back next to oil pump in a centre part ( pos. 1);

- in the area of axial joint plane of compressor and gas turbine (pos. 2);

- next to the speed-reducer/gear unit (box) (pos. 3);

- next to the air press (4 and 5 pos.).

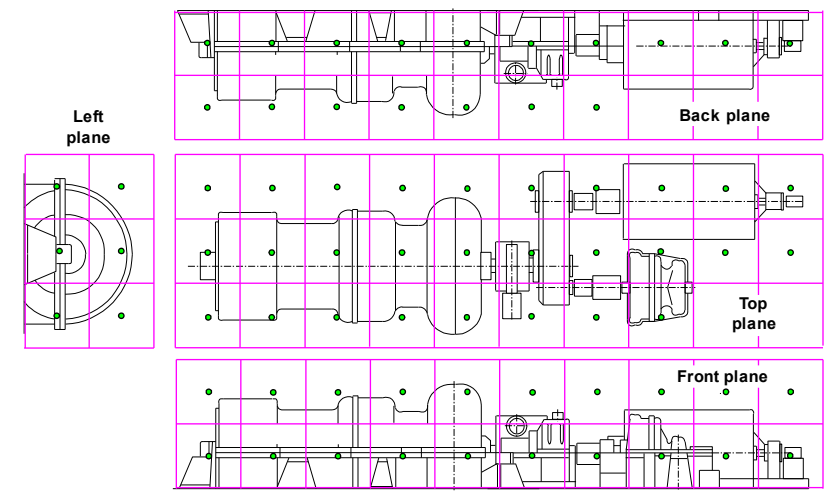

Fig. 7 Involute of turbo compressor's measured surface (without the right plane)

On the base of the results obtained after investigation of noise nature, the acoustic screen material was selected and they covered the maximum noise emission zones. The results of such noise reduction physical modelling are presented in Fig. 8. 


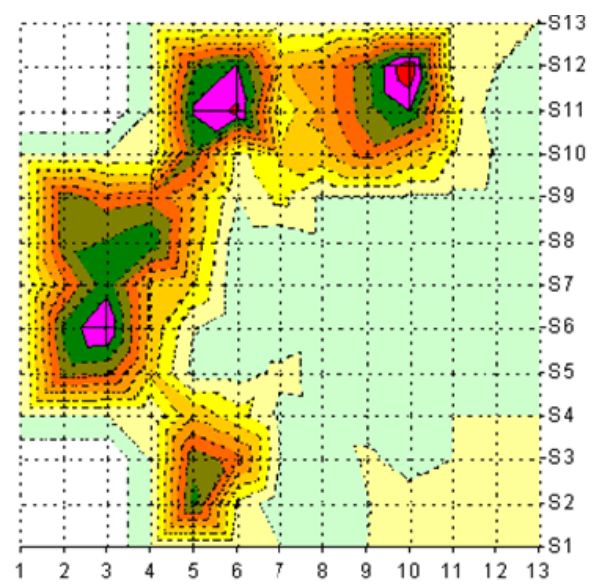

a

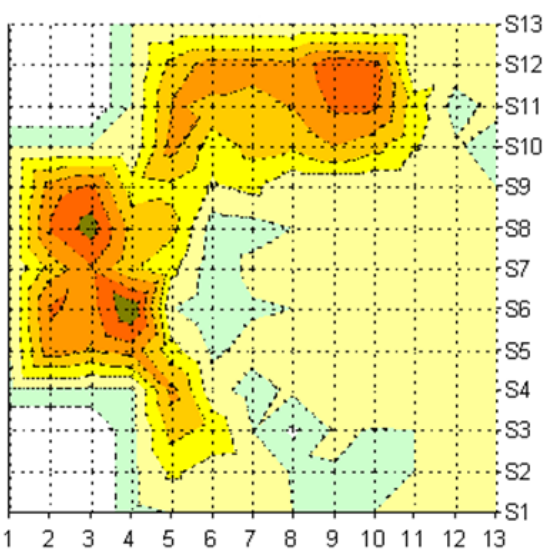

b

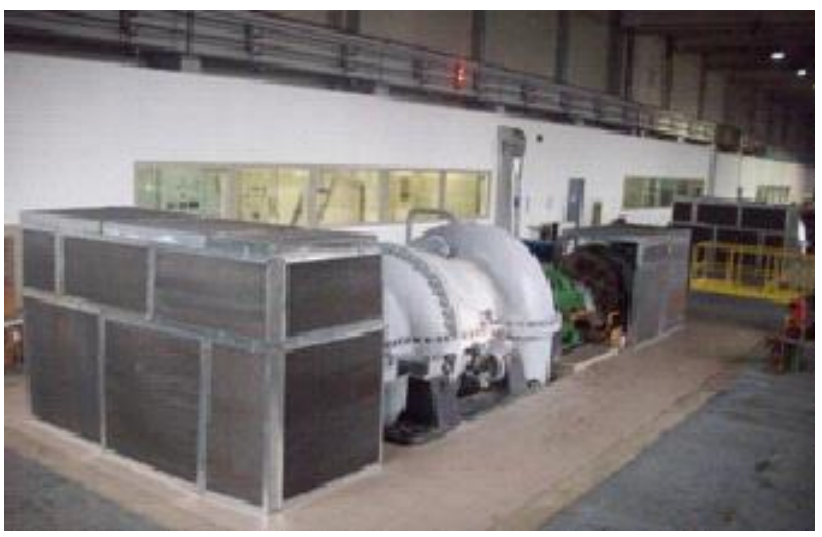

$\mathrm{c}$

Fig. 8 The maps of turbo compressor's emitted noise sound intensity (a) and pressure (b) variations, when the zone with the maximum noise emission next to the axial compressor is covered with sound absorbing plates (c)

The provided results show that by covering the area of the turbo compressor zone next the axial compressor with sound absorbing plates, the acoustic noise level is significantly reduced in that zone. It is possible that by covering all eight separate zones of turbo compressors with optimal size easily removable acoustic screens will reduce total acoustic noise level in the machine premises.

The performed investigation shows that acoustic fields can be reduced by using passive means made of sound absorbing material. Acoustic material must feature particular characteristics, which are determined by using sound noise measurement spectral analysis and physical modelling. For appropriate and optimal use of the means in particular technical environment the results provided in this study are essential. If the experiments require a lot of resources and the obtained information is insufficient to make conclusions about the characteristics of the technical environment - it is possible to apply mathematical modelling. In this case there is a need to choose the assumptions which would ensure the adequacy of the models for the real acoustic fields. The model identification by using physical experiment data might be useful.

\subsection{Mathematical modelling of acoustical fields}

In order to reproduce adequately the acoustic field in technical environment, the acoustic excitation modelling is very important. The variety of maintained equipment in technical environments both in the sense of frequency spectrum and emitted power requires separate investigation of separate source acoustic parameters. In this study the noise emitted by maintained turbo compressor was modelled (Fig. 2, a). For this purpose when measuring sound intensity according the above described methodology the zones in the turbo compressor where the maximum noise was emitted (extreme zones). Similar noise measurements were made for all eight turbo compressors exploited in compression section of the machine room.

The data obtained from the turbo compressor emitted noise were used for theoretical 2D sources model by using finite elements method (FEM). The excitation source was modelled by distinguishing five circular zones, their size the same as extreme zones of the turbo compressors: axial compressor, gas turbine, reducer and air press. In these zones specific values for acoustic pressure were set. It is known [18], that intensity which characterizes the directivity of acoustic power and sound pressure are related:

$$
I=\frac{W}{S}=\frac{p^{2}}{\rho c}
$$

here $I$ is sound pressure intensity, $\mathrm{W} / \mathrm{m}^{2} ; W$ is acoustic power, $\mathrm{W} ; S$ is surface area through which during particular time acoustic power permeates, $\mathrm{m}^{2} ; p$ is sound pressure in specific point; $\rho$ is air density, $\mathrm{kg} / \mathrm{m}^{3} ; c$ is the speed of sound wave, $\mathrm{m} / \mathrm{s}$. The sound pressure values had to be set such that acoustic power emitted by every separately modelled turbo compressor and its directivity would comply with the values determined experimentally. For this purpose optimisation analysis was performed with objective function of turbo compressors emitted acoustic power, state variables - acoustic intensity in front, back, left and right plane directions, designed variables - sound pressure values in extreme zones. The optimization analysis was performed using subproblem approximation method [19]. Thus by using penalty functions the minimizing is performed:

$$
F\left(\bar{x}, p_{k}\right)=\hat{f}+f_{0} p_{k}\left(\sum_{i=1}^{n} X\left(x_{i}\right)+\sum_{i=1}^{m_{1}} R\left(\hat{r}_{i}\right)+\sum_{i=1}^{m_{2}} B\left(\hat{b}_{i}\right)+\sum_{i=1}^{m_{3}} L\left(\hat{l}_{i}\right)+\sum_{i=1}^{m_{4}} G\left(\hat{g}_{i}\right)\right),
$$


here $F\left(x, p_{\mathrm{k}}\right)$ is unconstrained objective function (also termed a response surface); $\bar{x}$ is design variable vector; $p_{\mathrm{k}}$ is a response surface parameter; $f_{0}$ is reference objective function value; $\hat{f}$ is approximated objective function; $X$ is penalty function of the designed variable; $R, B, L$ and $G$ are penalty functions of state variables, in front, back, left and right planes directions, respectively; $\hat{r}_{i}, \hat{b}_{i}, \hat{l}_{i}, \hat{g}_{i}$ are state variables, acoustic intensity in the front, back, left and right plane directions, respectively (Fig. 1, b). The optimization analysis led to obtaining values of designed variables, i.e. sound pressure in the extreme zones of every turbine.

The adequacy of the modelled excitation source was tested experimentally. During the experiment the acoustic field of the maintained turbo compressor was measured without sound absorbing screens and when max imum noise emission zone next to axial compressor is covered with screens and sound intensity in four directions is calculated. The division of measured surface into segments is presented in Fig. 7.

The values of sound intensity level, measured experimentally and calculated using theoretically modelled excitation source, using FEM model in four directions is provided in Fig. 9.

The obtained results of the modelled noise source acoustic field show that by applying mathematical modelling the adequate reconstruction of exploited turbo compressor's acoustic field sound intensity and evaluation of its acoustic power are possible when the sound absorbing screens are used. By comparing sound intensity level for different cases: with sound absorbing planes and without them it can be seen that in first case it was reduced in left and backplane direction by $14 \%$.

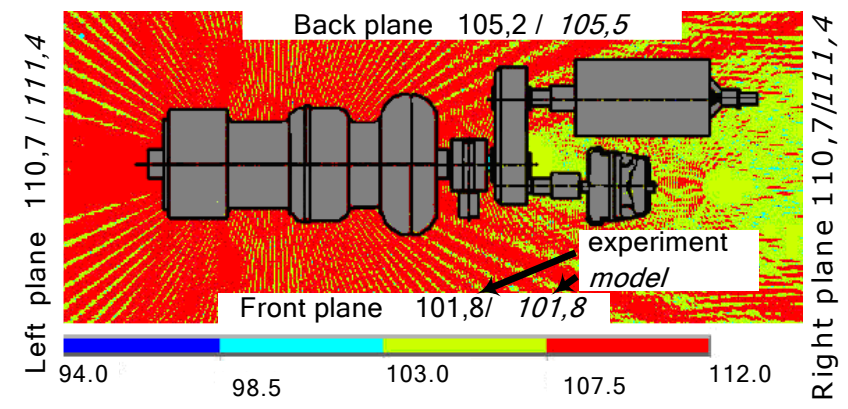

a

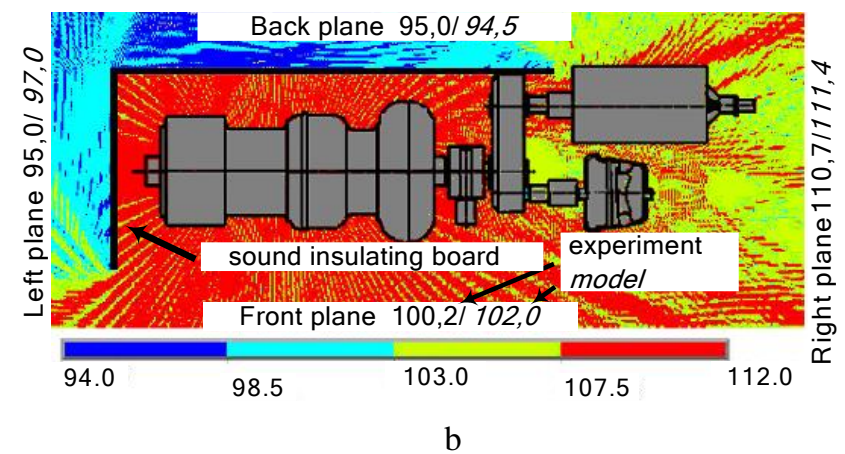

Fig. 9 Sound pressure level calculated using FEM in the environment of the a - uncovered turbo compressor; $b$ - turbo compressor partially covered with sound absorbing screens; sound intensity (dB) determined experimentally and using modelling in different directions

\subsection{Evaluation of efficiency of methodology application}

The efficiency of suggested methodology was executed according the decrease of $L_{A e q}$ and correspondingly increase of $T_{e}$. Fourteen acoustic screens arrangement model were tested totally. Their main feature was that by optimizing arrangement of the screens the minimal sound pressure level next to one of idle (being repaired) compressor. In practice it was accomplished by using procedure presented in Fig. 10. The Fig. 11 summarizes the results of methodology realization prototype model according the decrease of $L_{A e q}$ and correspondingly the increase of $T_{e}$.

The tendency of increment of prototype model efficiency was obtained by comparing $L_{\text {Aeq }}$ decrease and respective $T_{e}$ increase with initial noise level in compression room turbo compressors hall. It shows that all the means increase efficiency, but the influence of the means is different. The biggest influence is obtained by covering compressors' air presses and axial compressors with casings made of ISOTEC KVL-100 acoustic panels and covering the floors. Other applied means did not cause any significant changes in the values criteria.

The efficiency of technology was evaluated by comparing with alternative noise reduction technology by using absorbance screens, often used in practice $[4,8]$. The analysis shows that in order to reach the result of the prototype ( $L_{\text {Aeq }}$ reduction from $110 \mathrm{dBA}$ to $95 \mathrm{dBA}$ ) in case of alternative technology very big investment is required (30...40 times larger than suggested technologies). Moreover, by applying some other suggested technological means, the efficiency would increase and $\mathrm{L}_{\text {Aeq }}$ decrease even more and in such case the area of the whole shop machine hall ceilings and walls is not sufficient to apply absorbance screens used in alternative technology.

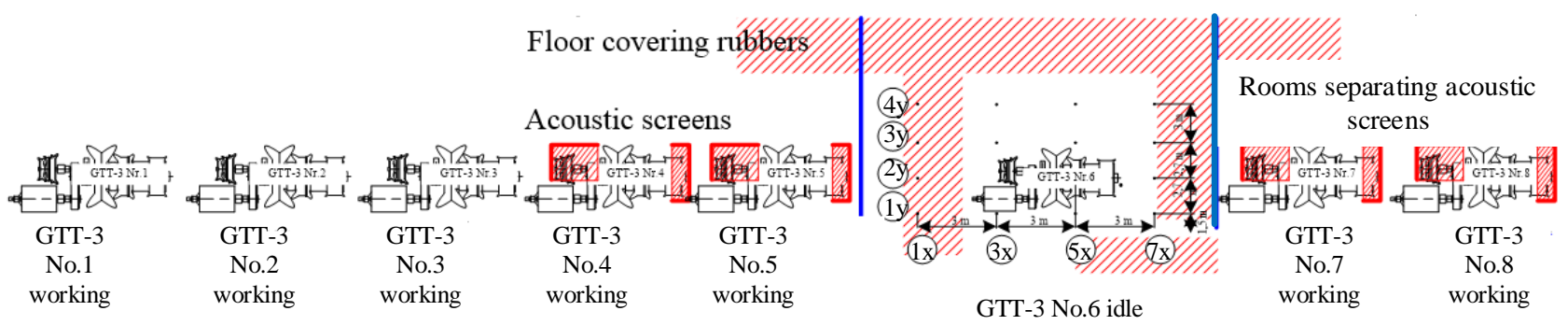

Fig. 10 The (real) means for the third model optimization according the acoustic fields control technology prototype: combination of screens and floor covers 


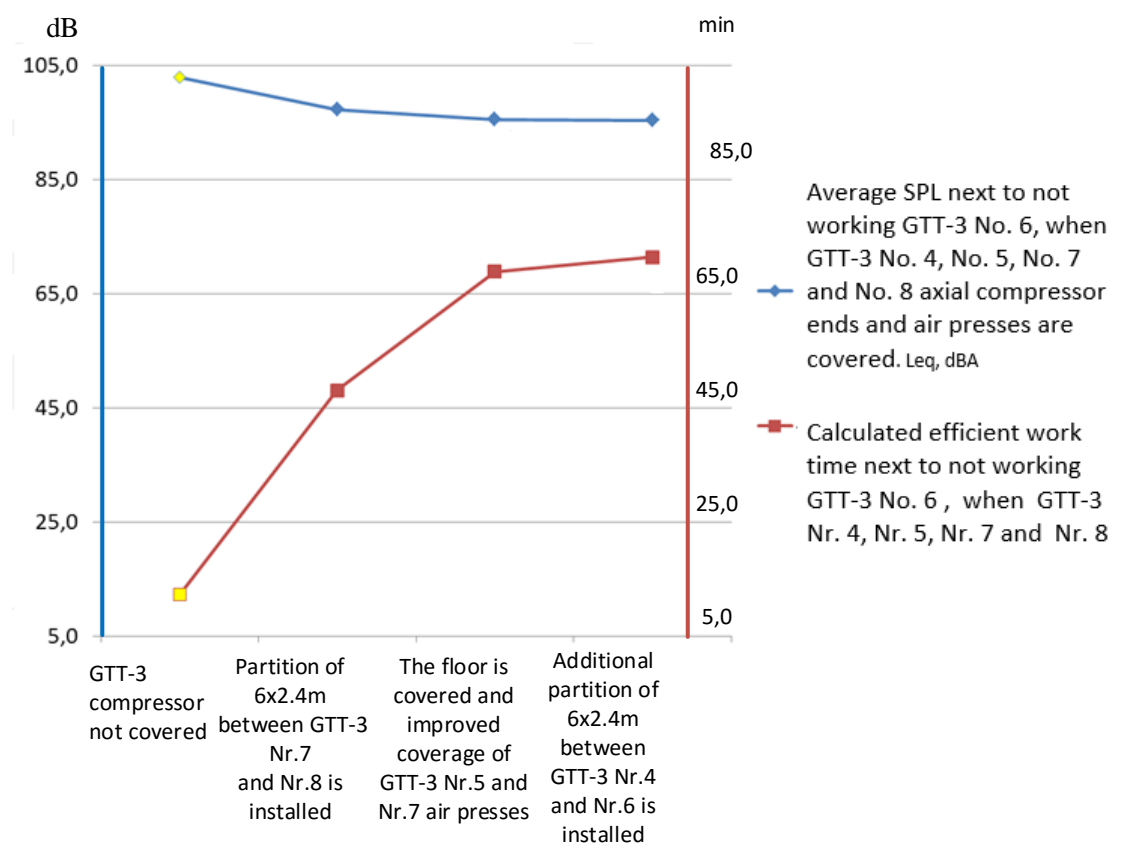

Fig. 11 Trend of increment of methodology prototype model efficiency

\section{Conclusions}

The executed investigations allowed creating strategy for reduction of noise emitted in closed space by several sources and approve its elements. The main idea of the suggested strategy - by using passive acoustic screen identify and acoustic separate the zones of aggregate which emitted sound in machinery hall in shop's compression section and thus reduce general sound pressure level. There were noise reduction models suggested and corresponding practical cases for noise level optimization technology.

For the optimization the most efficient - the third technology prototype (Fig. 11) realization model was selected as its optimization need and efficiency of actions can be evaluated according the increment of efficient work time $T_{e}$ :

- for non-optimized prototype of the third model the $T_{e}$ increased 4 times;

- for optimized prototype of the third model - 6 times.

When comparing suggested technology with widely used absorbance screens technology [7] the effect is as follows:

- the current sound pressure level decrease effect (in repair zone) was achieved with 40 times lesser investments for acoustic materials than covering shop ceilings and walls with acoustic panels (the sound pressure reduction method by using absorbance screens);

- my applying the above mentioned method even by covering the whole area of the shop ceiling and walls with absorbance screens, the sound pressure level decreases only to $94 \mathrm{~dB}$.

\section{Reference}

1. Hygiene norm HN 30:2009 "Infrasound and low frequency sounds: Limit values for residential and public buildings". Lithuanian hygiene norm.

2. Jakobsen, J. 2012. Danish regulation of low frequency noise from wind turbines, Proceedings of the confer- ence on Low Frequency Noise, Stratford upon Avon, UK, $22-24$.

http://dx.doi.org/10.1260/0263-0923.31.4.239.

3. Hygiene norm HN 33:2011 "Noise limit values for residential and public buildings and they surrounding", Lithuanian hygiene norm.

4. Barron, R.F. 2003. Industrial Noise Control and Acoustics, New York: Marcel Dekker Inc., 552 p.

5. Cavalieri, A.V.G.; Daviller, G.; Comte, P.; Jordan, P.; Tadmor, G.; Gervais, Y. 2011. Using large eddy simulation to explore sound-source mechanisms in jets, Journal of Sound and Vibration 330(17): 4098-4113. http://dx.doi.org/10.1016/j.proeng.2010.09.012.

6. Catana, D. 2013. Study regarding industrial noise reduction with sound absorbing screens, Bulletin of the Transilvania University of Braşov, Series I: Engineering Sciences 6(55) No. 1: 12-18.

7. Mikalauskas, R.; Volkovas, V. 2009. Modeling of sound propagation in the closed space and its interaction with obstacles, Mechanika 80: 42-47.

8. Stauskis, V.J. 2005. Structural Acoustics (in Lithuanian), Vilnius: Technika. 268p.

9. Night noise guidelines for Europe. 2009. World Health Organization (WHO), Copenhagen.

10. Oliva, D.; Hongisto, V.; Keränen, J.; Koskinen, V. 2011. Measurement of low frequency noise in rooms. Indoor Environment Laboratory, Turku, Finnish Institute of Occupational Health, Helsinki, Finland.

11. Ministry of the Environment Government in Japan. 2004. Handbook to deal with low frequency noise, Japan.

12. Von Estorff, O. 2011. Modern numerical methods to solve real life acoustics problems, Proceedings Acta Acusticum united with Acustica, 17-18 p.

13. Pedersen, S.; Møller, H.; Persson Waye, K. 2007. Indoor measurements of noise at low frequencies problems and solutions, J. Low Freq. Noise Vibration and Active Control 26(4): 249-270. http://dx.doi.org/10.1260/026309207783571389.

14. Roozen, N.B.; Van den Oetelaar, J.; Geerlings, A. 
2009. Source identification and noise reduction of a reciprocating compressor; A case history, International Journal of Acoustics and Vibration Vol. 14(2): 90-98.

15. International Organization for Standardization ISO 11690-1. 1996. Acoustics - Recommended practice for the design of low-noise workplaces containing machinery - Part 1: Noise control strategies.

16. International Organization for Standardization ISO 11690-1, Acoustics - Recommended practice for the design of low-noise workplaces containing machinery Part 2: Noise control measures, 1996.

17. International Organization for Standardization ISO 11690-1. 1997. Acoustics - Recommended practice for the design of low-noise workplaces containing machinery - Part 3: Sound propagation and noise prediction in workrooms.

18. Beranek, L.L. 1996. Acoustics. New York: Sunnyside Blvd., Woodbury. 255p.

19. ANSYS, Release 13.0. 2010. User's manual, US.
R. Mikalauskas, V. Volkovas

\section{PRACTICAL ASPECTS OF INCREASING WORKERS EFFICIENT WORK TIME IN A HIGH NOISE LEVEL INDUSTRIAL AREA}

\section{S u m m a r y}

The acoustic noise problem of industrial areas widely investigated and discussed. It considered as important while ensuring human safety and health. In the paper, we suggest to evaluate the impact of technical environment to the workers by investigating acoustic fields and adequately controlling them. These fields, generated by various sources, usually characterized by specific features, making their investigation and control a difficult problem, where only complex methods can be effectively applied. In systematic point of view, common methodology suggests for investigating and controlling specific acoustic fields in a closed space. The practical aspects analyzed for a particular case of compressor room acoustic noise reduction and increase of efficient work time for workers in a high-level noise technical environment.

Key words: workplace's acoustic noise, control methodology, field modelling, noise reduction.

Received September 29, 2015 Accepted November 12, 2015 